\title{
DISCUSSION (Hubený)
}

MICHAUD: Is there a systematic effect of NLTE on line blanketing due to type $C$ effects dominating for many ions?

HUBENY: Yes, such an effect may exist, and it will depend on $T$ eff and abundance. For example, in AO stars, if $\mathrm{Fe}$ II is the dominant source of blanketing opacity, the type $\mathrm{C}$ effect is not large for $\mathrm{Fe}$ II, but it may be large for $\mathrm{Fe} I$, which would be underpopulated. Thus, the overall blanketing in models is not affected very much for AO stars. The situation is different for hotter stars, where the balance between $\mathrm{Fe}$ III and $\mathrm{Fe}$ II shifts towards the dominance of $\mathrm{Fe}$ III.

These statements should be verified by calculating NLTE line blanketed model atmospheres for A and B stars. A step in this direction has recently been taken by Anderson (Ap. J., in press), who has calculated NLTE models of hot stars ( $T_{f f} \approx 35000 \mathrm{~K}$ ) taking into account blanketing effects. He has found a number of interesting phenomena.

There is hope that similar calculations will be performed for cooler stars soon. For example, in peculiar A stars, if rare earths are the dominant source of blanketing opacity, singly ionized rare earths surely are affected by type $C$. One needs always to bear in mind the type of star and the dominant source of blanketing.

ROMANOV: What is your opinion about the influence of the conditions of ionization on the determination of the abundance of elements in hot stars?

HUBENY: I think you are referring to the decrease of the ionization potential? Yes? I haven't made calculations, but it seems to me that those effects should be less than possible NLTE effects. The lowering of ionization potential is already included in the partition functions, even in LTE calculations, and NLTE calculations also take this into account. Comparing LTE calculations with and without lowering, and NLTE with LTE calculations taking lowering into account, which I think is the basis of your question, the answer depends on the ion. For those ions in which type $C$ effects will occur, almost certainly NLTE effects will dominate. There may be some special situations in which the effect of lowering might be important, but to my knowledge this has not yet been systematically studied.

STEPIEŃ: I think that it is generally accepted that if you have significant lowering of ionization potentials this is due to collisions playing a larger role, so the departures from LTE are generally smaller for small ionization potentials. It is the same for higher Iine excitation levels.

HUBENY: This is an important problem, because there are two approaches to the question: how do you approximate the higher lying levels?. One way is to approximate the departure coefficient for the higher levels by the same coefficient used for the highest level explicitly included in the calculation. This approach has been adopted by Borsenberger, Michaud and Praderie in their diffusion calculations, and essentially says that coupling with the continuum is comparatively less than coupling with lower lying levels. On the contrary, one may very reasonably assume that high lying levels are strongly coupled with the continuum. so that departure coefficients would be more or less equal to 
unity. To decide which approximation is more appropriate is very difficult, because the detailed form of the collision rates for those levels is, unfortunately, not very well known.

For example, in He I, the departure coefficients for levels with $\mathrm{n}=5$ are very similar to levels with $\mathrm{n}=4$ and nowhere near unity due to collisional coupling. So, this argument that very high lying levels must be near to LTE might not be quite correct in many situations. STĘPIEN: Yes, I agree.

DROBYSHEVSKI: I wonder why you did not discuss effects of magnetic fields on NLTE. I mean not the atomic level splittings and other atomic effects, but so called gas discharge phenomena, with strong differences between electronic and ionic temperatures, particle beaming, laser effects, etc.

HUBENÝ: I have deliberately omitted these phenomena and concentrated on the "classical" stellar atmospheres problem (plane-parallel stratification, hydrostatic and radiative equilibrium). From the methodological point of view, I feel that it is necessary first to understand well the simpler situations before attempting to treat more complicated configurations. The primary question is: to what extent will a physically more consistent description of the interaction of radiation with matter affect the methodology of interpretation of observed stellar spectra? As I have shown, this problem is still far from being solved. Of course, there are large effects of magnetic fields on hydrostatic equilibrium, and so on, and these need to be studied.

STEPIEN: So you are not against magnetic fields!

HUBENÝ: Not at all!

DWORETSKY: In some cases an observer can see three stages of ionization of an element in the spectrum of a normal or peculiar star simultaneously. Given that the atomic data are good, and that (usually) the lines seen are due to resonance transitions, then if an LTE analysis gives the same abundance for the element for all three stages, can we actually have confidence that the result is correct?

HUBEN Y: Well, this may be a strong argument in favour of the reliability of the result, but strictly speaking one can not definitely rule out the possibility that some unexpected physical phenomena produced a spurious interpretation of observations. In this respect, an interesting example is provided by an analysis of the C II resonance lines in Vega. The profiles synthesized assuming LTE and NLTE give the best fit for carbon abundances $3 \cdot 10^{-4}$ and $2 \cdot 10^{-4}$, respectively (Friere, Astr. Astrophys., 78, 148, 1979). The NLTE result was obtained without partial redistribution. My own result, for NLTE and partial redistribution, gives the value $3 \cdot 10^{-4}$, the same as the LTE calculation. This was purely accidental! Yet one can not conclude that the LTE approach is correct and NLTE incorrect. This does not answer your question, but shows that one should check very carefully whether the diagnostic method has not neglected some important physical phenomena which may spoil the interpretation. Generally, NLTE releases us from logical binds into which we may fall when trying to interpret observations solely on the basis of LTE.

STEPIEN: So, I think the conclusion is, Dr. Dworetsky, that your abundance may accidentally be correct! [laughter] 
ALECIAN: One must also remember that abundance stratifications of some elements may affect strongly the radiative transfer in the lines. The abundances deduced for these elements by classical methods must be interpreted differently if these elements are strongly stratified (above, $\tau_{5000}=10^{-1}$ ).

HUBENÝ: I completely agree with you. Some situations require a detailed NLTE analysis.

STEPIEF: When an element is pushed up in an atmosphere and is collected in a thin layer at the top, NLTE effects can be more pronounced than in the case of a uniform distribution. Would you expect to find any observable differences between these two cases and could you obtain a criterion to distinguish between a uniform and concentrated distribution of the element?

HUBENÝ: Differences between both cases do certainly exist, but they need not necessarily be easily observable. Each particular case requires careful study. Very few calculations of this sort have been made so far, and it is very difficult to make any quantitative (or qualitative) predictions. 